\title{
A relação entre a mulher com diabetes e o seu corpo: o risco da diabulimia
}

\author{
The relationship between women with diabetes and their body: the risk of diabulimia \\ La relación entre mujeres con diabetes y su cuerpo: el riesgo de diabulimia
}

\author{
Letícia Braga Ribeiro ${ }^{1}$ (b) \\ Claudia Maurício Pieper ${ }^{2,3}$ (D) \\ Giovana Andrade Frederico ${ }^{1}$ (i) \\ Mônica Antar Gamba ${ }^{1}$ (D) \\ Anderson da Silva Rosa ${ }^{1}$ (1)
}

1. Universidade Federal de São Paulo, Escola

Paulista de Enfermagem. São Paulo, SP, Brasil.

2. Pontifícia Universidade Católica do Rio de Janeiro. Rio de Janeiro, RJ, Brasil.

3. Instituto Estadual de Diabetes e

Endocrinologia Luiz Capriglione. Rio de Janeiro, RJ, Brasil.
Autor correspondente:

Giovana Andrade Frederico.

E-mail: giovanaafrederico@gmail.com

Recebido em 26/07/2020.

Aprovado em 29/01/2021.

DOI:https://doi.org/10.1590/2177-9465-EAN-2020-0270

\section{RESUMO}

Objetivo: compreender a relação que as mulheres com diabulimia têm com o corpo. Método: pesquisa qualitativa, desenvolvida com o Método da História Oral Temática, com quatro mulheres entre 18 e 30 anos que autorreferiram diabulimia. Resultados: a relação das mulheres com o seu corpo foi pautada pela pressão social de magreza feminina que distorce a autoimagem, gera insatisfação com o corpo e a diminuição da autoestima. A perda de peso é proporcionalmente associada ao aumento da aceitação social e justifica a utilização de métodos deletérios à saúde como a diminuição da dose ou a suspensão do uso de insulina As narradoras demonstraram conhecer os riscos, mas admitiram repeti-la em determinados contextos. Considerações finais e implicações para a prática: o desejo que as mulheres com Diabetes Mellitus têm de perder peso precisa ser incorporado às estratégias de cuidado com a atenção dos profissionais da saúde à subjetividade, atuando para reconhecer e prevenir os transtornos alimentares em mulheres com diabetes e minimizar a adoção de ações prejudiciais à saúde.

Palavras-chave: Diabetes Mellitus; Transtornos da Alimentação e da Ingestão de Alimentos; Bulimia Nervosa; Cetoacidose Diabética; Enfermagem.

\section{Abstract}

Objective: to understand the relationship that women with diabulimia have with the body. Method: a qualitative research, developed with the Thematic Oral History Method, with four women between 18 and 30 years of age who reported diabulimia. Results: women's relationship with their bodies has been guided by the social pressure of female thinness that distorts self-image, generates dissatisfaction with the body and diminishes self-esteem. Weight loss is proportionally associated with increased social acceptance and justifies the use of harmful methods to health such as reducing the dose or suspending the use of insulin. The narrators showed to know the risks, but admitted to repeat it in certain contexts. Final considerations and implications for practice: the desire that women with Diabetes Mellitus have to lose weight needs to be incorporated into the strategies of care with the attention of health professionals to subjectivity, acting to recognize and prevent eating disorders in women with diabetes and minimize the adoption of actions harmful to health.

Keywords: Diabetes Mellitus; Eating Disorders and Food Ingestion; Bulimia Nervosa; Diabetic Cetoacidosis; Nursing

\section{RESUmen}

Objetivo: comprender la relación que tienen las mujeres con diabulimia con el cuerpo. Método: investigación cualitativa desarrollada con el Método de Historia Oral Temática, con cuatro mujeres entre 18 y 30 años que autoinformado diabulimia. Resultados: la relación de la mujer con su cuerpo estuvo guiada por la presión social de la delgadez femenina que distorsiona la imagen de sí misma, genera insatisfacción con el cuerpo y la disminución de la autoestima. La pérdida de peso se asocia proporcionalmente con una mayor aceptación social y justifica el uso de métodos nocivos para la salud, como reducir la dosis o suspender el uso de insulina. Los narradores demostraron conocer los riesgos, pero admitieron repetirlo en determinados contextos. Consideraciones finales e implicaciones para la práctica: el deseo que tienen las mujeres con Diabetes Mellitus de adelgazar debe incorporarse a las estrategias de atención con la atención de los profesionales de la salud a la subjetividad, actuando para reconocer y prevenir los trastornos alimentarios en mujeres con diabetes y minimizar la adopción de acciones nocivas para la salud.

Palabras clave: Diabetes Mellitus.; Transtornos de Alimentación y de la Ingestión de Alimentos; Bulimia Nerviosa; Cetoacidosis Diabética; Enfermagem. 


\section{INTRODUÇÃO}

O Diabetes Mellitus (DM) é uma condição crônica que cursa com várias complicações clínicas e está entre as maiores causas de morbidade e mortalidade no Brasil e no mundo. Estima-se que, mundialmente, o DM afete, aproximadamente, 463 milhões de pessoas com idades entre 20 e 79 anos, sendo que mais de $87 \%$ das mortes ocorrem em países de baixa e média renda, provavelmente, em virtude de vulnerabilidades sociais que geram desigualdades no diagnóstico oportuno e no acesso aos cuidados de saúde, rede de apoio e tratamentos adequados em situações especiais. Estima-se que, no mundo, 232 milhões de pessoas ainda não foram diagnosticadas com o DM. Dentre os países, o Brasil é o quinto país em número de pessoas com diabetes, com aproximadamente 16,8 milhões de pessoas com idades entre 20 e 79 anos com o Diabetes Mellitus tipo 2 (DM2). ${ }^{1}$

Crianças e adolescentes convivem com o Diabetes Mellitus tipo 1 (DM1) e estes são cerca de 1,1 milhão no mundo, ${ }^{1}$ tornando essencial a implementação de políticas públicas específicas a este grupo que contemplem não apenas o tratamento medicamentoso e a prevenção de complicações no corpo, mas o cuidado nas dimensões social e psicológica e suas repercussões nas famílias.

A partir de uma revisão sistemática, foi possível identificar a associação entre a vitimização por bullying e a presença de DM1 na comparação entre pessoas vivendo com ou sem condições crônicas. O reconhecimento das situações de violência, de diversas naturezas, desde a primeira infância, torna primordial o desenvolvimento de estratégias para o acompanhamento das crianças e jovens e que visem à conscientização da sociedade, às relações interpessoais saudáveis e à promoção da saúde. ${ }^{2}$

Há evidências da relação entre a pessoa com DM e comorbidades psiquiátricas, principalmente depressão e Transtornos Alimentares (TAs), podendo interferir no controle metabólico e aumentar as complicações do diabetes. Dentre os vários fatores associados entre o DM e os TAs, os aspectos psicológicos relacionados ao diabetes, assim como as restrições dietéticas, desempenham função importante na expressão de comportamentos alimentares inadequados nessa população. . $^{3,4}$ Estudos apontaram como fatores de risco o sexo feminino, o sobrepeso/obesidade, a omissão ou a redução de insulina e a insatisfação com o corpo. ${ }^{5}$

Destaca-se que adultos jovens com condições crônicas de saúde relacionadas à dieta, como o DM, são mais propensos ao desenvolvimento de distúrbios alimentares, com comportamentos compensatórios para manter ou reduzir o peso corporal como a adoção de práticas esportivas em excesso e/ou o uso de medicamentos. Essas pessoas também tendem a apresentar mais sintomas depressivos e de ansiedade, se comparadas com as pessoas sem condições crônicas. ${ }^{6}$

Estudo envolvendo 282 pessoas com DM1, com idades $\geq 18$ anos, identificou que os transtornos da alimentação e da ingestão de alimentos, a depressão e a ansiedade eram mais prevalentes em mulheres, especialmente adolescentes e jovens. Os TAs foram identificados em $20,3 \%$ dos participantes, enquanto a depressão e a ansiedade estiveram presentes em
$6,2 \%$ e $19,0 \%$, respectivamente, alertando os profissionais da saúde, especialmente da atenção primária à saúde, sobre a necessidade da atenção interdisciplinar para além do tratamento medicamentoso. $^{7}$

Práticas como a redução ou omissão do uso de insulina por pessoas com DM1 têm sido reconhecidas como diabulimia. Estão associadas à preocupação com o peso e a imagem corporal, a aceitação pessoal e da sociedade, gerando maior satisfação com a imagem corporal e a autoestima. ${ }^{8}$

A redução ou a supressão da insulina acarreta hiperglicemia, poliúria e alterações metabólicas que produzem, como consequência, a redução do peso corporal. Em detalhe, tecidos sensíveis à insulina, como não conseguem utilizar a glicose, passam a metabolizar principalmente gorduras em vez de carboidratos, acarretando quadros graves de cetoacidose diabética com necessidade de internação hospitalar. Como a insulina é um hormônio anabólico, sua deficiência favorece processos catabólicos como lipólise, proteólise e glicogenólise. A redução de peso causada por esse processo pode vir acompanhada de desidratação, náuseas, êmese, além de complicações microvasculares devido ao diabetes e o risco de morte. ${ }^{9} \mathrm{~A}$ omissão da dose de insulina, por si só, pode causar o aparecimento precoce (quatro anos ou menos de doença) de complicações crônicas como a retinopatia, neuropatia e nefropatia. ${ }^{9,10}$

Devido aos potenciais agravos à saúde decorrentes da diabulimia e à sua maior incidência em mulheres jovens que relatam insatisfação com a imagem corporal, ${ }^{11}$ faz-se necessário estudar a construção social dessa doença e dos padrões de beleza que condicionam tais comportamentos.

O padrão atual de beleza é amplamente fortalecido pela mídia, responsável pela validação cultural e a objetificação humana, transformando padrões estéticos humanos em mercadorias extremamente caras e quase sempre inatingíveis para a grande maioria das pessoas. ${ }^{12} \mathrm{~A}$ chamada indústria da beleza gera lucros absurdos, atuando de diferentes formas, oferecendo desde produtos para dietas de baixas calorias, cosméticos, atividades físicas voltadas para a questão estética até o aumento progressivo de procedimentos cirúrgicos estéticos. ${ }^{13,14}$ Apesar das diferenças culturais e econômicas entre os países, o processo de globalização e o acesso instantâneo às informações tendem a unificar padrões de consumo relacionados à beleza, assim como ocorre com outras mercadorias disponíveis mundialmente.

A partir da construção social sobre o corpo feminino, as mulheres buscam formas de aprimoramento corporal para alcançar esse padrão, tanto para satisfazer sua autoestima quanto para a aceitação social. ${ }^{15}$ Emagrecer torna-se obrigatório e a obesidade torna-se negligência e irresponsabilidade da pessoa. ${ }^{14,16}$

Estudiosos ${ }^{17}$ argumentaram que a insatisfação com o peso está intimamente relacionada à ênfase cultural na magreza e ao estigma social atribuído aos que não se encaixam nesse padrão, principalmente entre as pessoas com obesidade. Nesse sentido, o padrão estético de beleza vigente e a excessiva preocupação da sociedade com uma imagem feminina muito magra são os principais fatores associados ao agravamento de TAs e às 
dificuldades advindas da obesidade, assim como ao aumento do nível de insatisfação corporal. ${ }^{18,19}$

Tal cenário precisa ser reconhecido pelos profissionais da saúde ao atender pessoas com diabetes. O risco para o desenvolvimento de TA pode ser precocemente detectado por meio de uma relação empática, solidária e colaborativa que permita a compreensão das angústias, sofrimentos e desejos individuais. ${ }^{20} \mathrm{~A}$ relevância dessa investigação concentra-se nos riscos de agravos à saúde ocasionados pela diabulimia.

Assim, este artigo tem por objetivo compreender a relação que a mulher com diabulimia tem com o seu corpo.

\section{MÉTODO}

Estudo com abordagem qualitativa, do tipo História Oral, na sua modalidade Temática, por corresponder a uma narração mais restrita do entrevistado acerca de um determinado tema. A História Oral devolve a história às pessoas em suas próprias palavras. E, ao Ihes dar um passado, ajuda-as, também, a caminhar para um futuro construído por elas mesmas. ${ }^{21}$

O Método da História Oral é construído por meio da conversa com pessoas sobre a experiência e a memória que cada um tem sobre fatos e valores que interessam ao pesquisador. Por meio da narrativa sobre uma realidade vivenciada, podem-se apreender as relações sociais mais globais. ${ }^{22} \mathrm{~A}$ presença do passado no presente imediato das pessoas é a razão de ser da História Oral. Nessa medida, ela não só oferece uma mudança do conceito da história, mas garante sentido social à vida de depoentes e leitores, que passam a entender a sequência histórica e sentemse parte do contexto em que vivem. ${ }^{23}$

A divulgação do projeto de pesquisa foi feita em um grupo de discussão na rede social FACEBOOK® denominado DIABETES E DIABULIMIA. No momento do recrutamento e seleção das participantes, o grupo contava com 266 mulheres. Os critérios de inclusão das participantes do estudo foram: ser mulher; ter idade entre 18 e 30 anos; autorreferir diagnóstico de diabetes e de diabulimia e participar do grupo de discussão DIABETES E DIABULIMIA.

As possíveis participantes foram informadas sobre os objetivos, a metodologia e as formas de divulgação da pesquisa e assinaram o Termo de Consentimento Livre e Esclarecido (on-line). As participantes que demonstraram interesse foram contatadas por mensagem pela pesquisadora principal e, posteriormente, as entrevistas foram agendadas. Não houve recusa em participação, no entanto, uma das possíveis entrevistadas não participou por incompatibilidade de horário e outra faleceu em decorrência do quadro de diabulimia antes da realização da entrevista. Assim compuseram este estudo quatro mulheres com DM1 e diabulimia.

As entrevistas foram desenvolvidas com roteiro semiestruturado contendo questões relacionadas à identificação pessoal e dados relacionados ao DM1, à relação com o próprio corpo e com o diabetes, à história relacionada ao uso de insulina, à visão e às atitudes da família e de profissionais da saúde quanto ao TA, ao histórico de complicações e sentimentos relacionados ao TA.
O projeto de pesquisa foi aprovado pelo Comitê de Ética em Pesquisa (CEP) da Universidade Federal de São Paulo, no dia 20 de maio de 2015, sob o Parecer nํㅜ 1.051.956/15. A coleta dos dados foi realizada pela técnica de entrevista pela autora principal após a aprovação do CEP. Os dados da pesquisa foram coletados entre maio a agosto de 2015.

As entrevistas presenciais foram realizadas com três participantes que residiam na cidade de São Paulo, Brasil, sendo uma na Universidade Federal de São Paulo e duas na avenida Paulista. Uma participante residia no Estado da Bahia, Brasil, e foi entrevistada via telefone. As entrevistas foram realizadas em um único encontro e tiveram entre uma hora e 30 minutos e duas horas cada. Salienta-se que foi proporcionado ambiente tranquilo, silencioso e reservado, não ocorrendo interferências nas entrevistas, que foram gravadas em arquivos de áudio.

Para a análise dos dados, aplicou-se o método da Análise de Discurso por meio dos seguintes passos: (1) ordenação dos dados obtidos por meio das entrevistas; (2) classificação dos dados obtidos nos textos (empíricos e teóricos), buscando relacionar as categorias analíticas (teóricas) e as empíricas; (3) reordenação das entrevistas, adotando o recurso utilizado na análise relacional; (4) realização de análise crítica relacional entre os dados empíricos obtidos nas entrevistas e as categorias analíticas selecionadas pela pesquisadora, buscando compreender e responder às questões elaboradas durante a fase de construção do objeto de estudo. ${ }^{22}$

O processo de análise deu origem a duas categorias analíticas: (1) A relação da mulher com o corpo, autoestima e padrões de sociais de beleza; (2) A relação das mulheres com a diabulimia e suas implicações.

O termo narradora foi escolhido para assegurar o sigilo dos nomes das mulheres que participaram do estudo.

\section{RESULTADOS}

\section{Participantes do estudo}

Narradora 1 (N1), 30 anos de idade, com DM1 há 13 anos, em uso de sistema de infusão contínua de insulina há nove meses, já recebeu orientação nutricional, porém, não aderiu, pratica atividade física, tem neuropatia periférica, é fisioterapeuta, casada, heterossexual e tem um filho.

Narradora 2 (N2), 27 anos de idade, com DM1 há 16 anos, em uso de insulina humana de ação intermediária (NPH) e insulina humana de ação rápida (regular), já recebeu orientação nutricional, porém, tem adesão que oscila conforme situações do dia a dia. Sedentária, tem neuropatia periférica, trabalha como assistente administrativo operacional, é solteira, heterossexual e não tem filhos.

Narradora 3 (N3), 19 anos de idade, com DM1 há oito anos, em uso de sistema de infusão contínua de insulina, já recebeu orientação nutricional, porém, sem segui-la em sua completude. Sedentária, sem complicações, é estudante de Enfermagem, solteira, heterossexual e não tem filhos. 
Narradora 4 (N4), 25 anos, com DM1 há 19 anos, em uso de insulina glargina e lispro, já recebeu orientação nutricional, porém, sem adesão. Sedentária, sem complicações, é estudante de moda, solteira, bissexual e não tem filhos.

\section{Relação das mulheres com o corpo, a autoestima e os padrões sociais de beleza}

Os resultados desta investigação revelaram que a relação dessas mulheres com o próprio corpo é conflituosa. Colocamse como reféns da imposição social de magreza feminina como sendo o único padrão de beleza aceito socialmente. Tal sujeição acarreta um estado crônico de insatisfação com o próprio corpo e um desejo intenso de emagrecer.

Olha, é muito importante, para mim, eu ter o corpo que eu desejo, é muito importante. (N1)

Bom, eu não me sinto bem com meu corpo porque, assim, hoje, tudo o que vale mais é a aparência. Não que eu esteja gorda, hoje, eu acho que eu sou magra demais e com uma barrigazinha assim meio, digamos, defeituosa. Eu como tudo integral: arroz integral; pão integral; manteiga light, gosto bastante dessas coisas. Mas acho que, se eu fizesse mais exercícios, se eu tivesse uma dieta mais rígida, quem sabe poderia até conquistar um certo tipo de corpo perto do que eu desejo. (N2)

Acredito que toda mulher sofra com o próprio corpo e tenha alguma coisa nele que gostaria de mudar. A diferença para nós, mulheres com diabetes, éque, quando se trata de peso, isso fica muito mais simples. (N4)

O processo de não aceitação da própria imagem corporal leva a uma diminuição da autoestima. Todas as narradoras apontaram a percepção de que estão acima do peso ou que suas formas corporais diferem dos padrões de magreza impostos pela sociedade, interferindo diretamente na autoestima. Tal percepção é também condicionada por situações em que a mulher precisa se expor e ser aceita socialmente.

Tem horas que isso me importa muito e tem horas que eu tô "cagando e andando" pra isso, então, varia muito. Tenho certeza de que, se a pressão social com corpo da mulher fosse menor, isso me afetaria menos porque a gente é muito julgada. Então, a opinião dos outros na nossa sociedade acaba sendo muito importante, então, tipo, ah, se você é taxada como gordinha ou como fora do padrão de beleza, isso mexe, pelo menos, comigo, mexe muito com a minha autoestima, então, sei lá, eu acho que é muito importante; se não tivesse essa pressão toda, se o gordinho fosse na moda, acho que todo mundo ia ficar, ia ser gordinho, entendeu, então, ninguém ia se importar com isso. (N3)

Meus problemas estavam muito mais relacionados com a minha autoestima do que com meu peso. (N4)

Para outra entrevistada, a motivação para alterar a imagem corporal foi relacionada a fatores externos, que influenciam a diminuição da autoestima, tais como: não se enquadrar no padrão de beleza veiculado pelas mídias; não encontrar roupas que sirvam adequadamente ou não estar inserida em um relacionamento amoroso. Tais situações foram apontadas como responsáveis pela recorrência do TA.

Eu diminuí bastante [a suspensão do uso de insulina], mas assumo que, uma vez na vida ou outra, eu ainda faço isso quando eu quero sair, quero vestir uma roupa que tá apertada, quando eu vejo que estou um pouquinho gorda, acima do peso e não tô conseguindo emagrecer, mesmo fazendo dieta e atividade física, então, eu vou lá e faço o mesmo processo. (N2)

A partir da insatisfação corporal e da consequente busca pelo corpo dentro dos padrões sociais, as mulheres com diabetes conseguem enxergar meios mais rápidos de atingir seus objetivos, apesar de toda a consequência negativa que isso possa ter para a sua saúde. Essa situação gera uma inversão de prioridades na qual a autoimagem passa a ser mais importante do que a própria condição de saúde dessas mulheres.

Eu já usei meu diabetes para emagrecer. Quando eu tinha 13 anos, eu comecei a não tomar insulina suficiente $e$ passar mal de propósito para começar a emagrecer, tipo, porque eu achava que isso tava sendo melhor do que cuidar do meu diabetes e continuar engordando. (N3)

\section{Relação das mulheres com a diabulimia e suas implicações}

Chamou a atenção, dentre as mulheres que participaram deste estudo, o fato de pensarem que apenas elas sabiam como utilizar do diabetes para conseguirem emagrecer.

Ela [médica] nunca me falou que isso existia, tipo nunca, sabe? Para mim, eu era a única que tinha feito isso na vida até meses atrás. Sei lá, pra mim, eu juro pra você, eu era a única que tinha ficado sabendo dessa tática da vida. (N3)

Uma vez, assim, de tanto fazer isso, eu comecei a pensar: será que só eu que faço isso? E fui pesquisar na internet e, quando coloquei lá no Google ${ }^{\circledR}$, apareceram várias pessoas, várias histórias com esse mesmo problema e foi aí que eu descobri até também o nome porque eu não sabia. (N2)

As mulheres tiveram dificuldade de definir com exatidão o momento em que começaram a diminuir a dose ou suspender o uso da insulina com o objetivo de emagrecer. Esse processo de manipulação do tratamento veio acompanhado de culpa pelo não cumprimento das orientações recebidas pelos profissionais e medo de interrupção da assistência recebida no serviço público.

Eu tinha medo de parar de tomar insulina. Rolava uma pressão muito grande pra eu controlar minha glicemia, já 
que, ao contrário dos médicos particulares, no hospital escola, eu era ameaçada de perder meu tratamento se não fizesse as coisas direito e isso também implicava em eu receber a insulina lispro, que era mais cara e o posto de saúde não fornecia. (N4)

Destaca-se o fato de o sentimento de culpa não estar relacionado aos possíveis prejuízos à saúde ocasionados pela suspensão do tratamento. Para outra narradora, o sentimento de culpa sequer existiu.

Eu nunca senti culpa por causa de ter feito isso, foi uma coisa assim, meio que natural. Quando eu percebi, eu já tava fazendo e, quando eu parei, eu parei e falei: Meu! Como assim? Não! Está errado, então, vamos fazer o certo. Ficar me sentindo culpada, remoendo, coisa assim, nunca aconteceu. (N3)

A motivação em cessar o uso da insulina foi evidenciada e relacionada à imagem corporal por uma das jovens.

Eu não consigo me desvencilhar deste desejo e, toda vez que eu me olho no espelho, me sinto motivada a parar novamente de tomar insulina. (N4)

Para além do diabetes e da preocupação com o próprio corpo, essas mulheres trabalham, estudam, têm duplas jornadas de trabalho e muitas outras responsabilidades. Tais fatores foram apontados como dificultadores para o autocuidado, pois nem sempre elas conseguem conciliar seus desejos e necessidades de cuidado com o ritmo de vida que levam.

O ritmo de vida que eu levo não me ajuda em nada cuidar do meu diabetes nem do meu corpo. É muito pesado, o ritmo que a gente tem na faculdade é muito corrido, não sobra tempo pra nada, muito menos pra cuidar da gente, e é muito difícil, a gente tem que se organizar. Essa coisa de ah, eu vou comer de três em três horas e vou fazer exame pré e pós-prandial, pô, meu! Você está no meio da aula, você não vai lembrar do pós-prandial e você não vai lembrar da comidinha de três horas depois. Sabe? Sei lá, é muito difícil ser regrada desse jeito. (N3)

Minha vida não se resume ao diabetes e nem a cuidar de mim, infelizmente. (N4)

\section{DISCUSSÃO}

A partir dos resultados e dos caminhos teórico-conceituais percorridos, apresenta-se uma possibilidade, dentre tantas outras, de compreender a diabulimia a partir da relação que as mulheres com diabetes estabelecem com o seu corpo. Vale ressaltar que a maioria dos estudos relacionados à temática de TAs, ${ }^{24}$ e especialmente da diabulimia, possui, como foco, a dimensão biológica do processo de adoecimento. As dimensões sociais e subjetivas precisam ser valorizadas para aumentar a potência dos cuidados oferecidos a essa população.

Os resultados deste estudo corroboram a literatura científica sobre a relação entre os TAs e as habilidades sociais em adolescentes ao apontar que o principal motivo para a insatisfação com a imagem corporal e a baixa autoestima nas mulheres está diretamente relacionado à pressão social em prol de um padrão de beleza feminino com a exaltação da magreza excessiva. ${ }^{18}$

As motivações de mudança da imagem corporal são derivadas de normas socioculturais que variam de acordo com o local e o período histórico e estão cada vez mais dinâmicas e globalizadas em decorrência da internet e das mídias sociais. Quem não se enquadra nos padrões de normalidade corre o risco de sofrer processos de exclusão social ${ }^{17,24}$ e pouco importa se tais padrões são distópicos e não condizentes para a maioria das pessoas.

Culturalmente, conceitos equivocados são construídos de que o sobrepeso é incompatível com a beleza e com a saúde. A pressão social contra o sobrepeso e a obesidade pode contribuir com diferentes níveis de distorção da imagem corporal. ${ }^{25}$ Esse contexto social que deturpa a autoimagem está ligado ao risco de desenvolvimento de TAs para a população em geral com incidência maior no gênero feminino jovem. ${ }^{8,25}$

A perda de peso é estimulada por diferentes mídias de comunicação e costuma ser valorizada como conquista em si sem levar em consideração que a saúde também se relaciona com o bem-estar e com o prazer associado ao comer. $\mathrm{O}$ resultado desse processo materializa-se na baixa autoestima, na insatisfação com o corpo e na disposição para a realização de dietas inconsequentes e que podem ocasionar agravos à saúde. Ao se tratar de mulheres jovens com DM1, o cenário é ainda mais preocupante.

Estudo de revisão, que teve como fonte primária blogs de pessoas com DM1 que relataram conviver com o fenômeno da diabulimia, confirmou resultados semelhantes aos deste estudo na medida em que identificou relatos de jovens que vivenciavam sofrimento exacerbado, informações sobre os motivos para a omissão do uso de insulina e estratégias de enfrentamento dessa importante condição de saúde pública mundial. Com restrição de evidências científicas, especialmente no Brasil, os relatos dos jovens tornam-se potentes aliados para o desenvolvimento e a promoção de intervenções ampliadas com a população. ${ }^{26}$

A promoção do cuidado e autocuidado em diabetes exige extrapolar a relação que se estabelece entre paciente $e$ profissionais de saúde. Sobretudo para crianças, adolescentes e suas famílias, a escola pode ser importante na ampliação de conhecimentos, na redução de preconceitos e estigmas, na construção de alternativas para minimizar as repercussões negativas que a doença pode ter na qualidade de vida, além de criar um ambiente favorável para a promoção da saúde física e emocional. ${ }^{27}$

Estudo de caso-controle apontou que 25\% das mulheres com DM1 apresentam TAs ao longo da vida, sendo que há uma tendência de aumentar a incidência desses transtornos mesmo após ao período correspondente à juventude adulta. ${ }^{28}$ 
Pessoas com diabetes e TAs ou sintomas de inadequação alimentar (síndromes subclínicas) apresentam um risco aumentado para desenvolver um pior controle glicêmico e, consequentemente, um maior risco de complicações agudas emergenciais e crônicas microvasculares irreversíveis pelo diabetes. ${ }^{3}$

Em um estudo de caso-controle com adultos jovens com e sem DM1 sob a perspectiva dos TAs e a insatisfação com a imagem corporal, foi possível avaliar os comportamentos, as atitudes e os sentimentos frente aos TA e suas percepções sobre a imagem corporal. Os resultados demonstraram que não houve diferença significativa entre os grupos, contudo, diversas alterações decorrentes do diabetes foram obtidas, como a influência na alimentação, na imagem corporal e no peso dos jovens. Essa tendência está relacionada a maiores taxas de morbidade e mortalidade para esse grupo e peso maior em relação ao grupo-controle. $O$ mesmo estudo também demonstrou a associação entre os TAs com o mau uso da insulina para a perda de peso. Foi relatado, por $7,3 \%$ dos participantes com controle glicêmico inadequado, o risco ao desenvolvimento de complicações microvasculares, sinalizando a necessidade de mais investigações e intervenções com esse público. ${ }^{29}$

No contexto brasileiro, um estudo evidenciou o risco aumentado de desenvolvimento de TAs em uma amostra de pacientes com DM $1 .{ }^{30}$ Esse risco foi associado ao sexo feminino, ao sobrepeso/obesidade, à redução de insulina ou à omissão e à insatisfação com o corpo. $O$ estudo apontou que a maioria das pessoas que omitiam o uso da insulina estava acima do peso e mais insatisfeita com sua imagem corporal. Esses resultados corroboram as narrativas das mulheres que participaram deste estudo. A insatisfação com o peso e a imagem corporal foi unanimemente apontada como estímulo para a manipulação do uso de insulina, mesmo tendo a consciência de que esse método de emagrecimento oferece riscos de agravos à saúde.

O fenômeno da diabulimia gera a restrição de insulina circulante (pela diminuição ou não aplicação da dose) e o acúmulo da glicose na corrente sanguínea promove a hiperglicemia constante e a excreção de glicose na urina, favorecendo a perda de peso, mesmo sem a adoção de cuidados alimentares..$^{30} \mathrm{~A}$ perda de peso é diretamente proporcional à elevação da glicemia. Assim, emagrecer, que era algo tão difícil, tornar-se-ia mais fácil. Apesar de existirem, muitas vezes, sintomas de cetose, isso ainda motiva as mulheres a permanecerem com esse comportamento, apresentando perda de peso e uma alimentação pouco saudável na qualidade e quantidade, entretanto, prazerosa.

Outro estudo brasileiro identificou uma prevalência elevada de adolescentes com DM1 e transtornos de saúde mental, com quase duas vezes mais que no grupo-controle. Os principais agravos identificados entre todos os participantes foram a depressão e a ansiedade e, ainda que com menos frequência, a presença de distúrbios alimentares. Também identificou que o peso em excesso eleva a probabilidade de os jovens desenvolverem transtornos mentais. Embora fosse alta a prevalência de adolescentes com problemas de saúde mental, apenas metade deles recebeu atendimento psicológico, e o acesso à avaliação psiquiátrica era ainda mais limitado, indicando lacuna no cuidado em saúde. ${ }^{31}$

Narrativas sobre formas de utilizar o diabetes para conseguir emagrecer e o pensamento das participantes de que apenas elas sabiam dessas possibilidades podem estar relacionados à pouca veiculação da bulimia nervosa, especialmente, em pessoas com diabetes, nas mídias populares brasileiras, apesar de ser um transtorno amplamente estudado em outros países. E, possivelmente, pela pouca valorização desse transtorno durante as orientações sobre os cuidados relacionados ao DM oferecidas pelos profissionais de saúde, culmina-se no agir silencioso, secreto e perigoso.

A detecção dos fatores de risco e a intervenção precoce são essenciais na tentativa da melhora e da manutenção da qualidade de saúde e bem-estar para crianças e jovens., ${ }^{3,29}$ Fazem-se relevantes uma comunicação aberta e colaborativa $e$ uma compreensão pormenorizada do usuário, individualmente, para identificar os comportamentos e intervir de forma adequada e eficaz.

Um estudo comprovou que as mulheres com DM1 eTAs não usam da mesma maneira outros métodos purgativos, como o vômito e laxantes, devido à facilidade da manipulação das doses de insulina para induzir a perda de peso. Também concluiu que a prevenção e o tratamento precoce de distúrbios alimentares em mulheres jovens com DM são importantes para reduzir a morbidade e a mortalidade em longo prazo decorrentes do controle metabólico inadequado e as complicações microvasculares. Essas mulheres podem apresentar características menos reconhecidas de um TA como o abandono do tratamento do diabetes, o controle metabólico instável, a hiperglicemia e a cetoacidose recorrentes, além do surgimento precoce de complicações microvasculares. Em comparação com as mulheres que são vistas em contextos psiquiátricos, as mulheres com diabetes e com distúrbios alimentares podem ter sintomas menos comuns e são mais propensas a ter um peso normal ou sobrepeso em vez de baixo peso. ${ }^{3}$

Diante da alta complexidade do tratamento do diabetes, torna-se necessária a associação medicamentosa com abordagens psicológicas..$^{32} \mathrm{~A}$ preocupação com os alimentos e a refeição, o gerenciamento do diabetes, juntamente com o risco de maior peso corporal, os sentimentos negativos sobre a forma do corpo e peso, a hiperglicemia crônica, a depressão, a ansiedade, a vergonha e o autocuidado deficiente compõem um cenário desafiador para o paciente e para o profissional da saúde no controle do quadro clínico. ${ }^{33}$

Ainda sobre as possibilidades de tratamentos, ${ }^{20}$ concluiu-se que o início da terapia com a bomba de insulina é associado com o endosso à diminuição de comportamentos de TAs em jovens com DM1. Porém, em pacientes que não aceitam o diabetes ou que possuam outras comorbidades psiquiátricas como a depressão, o uso de bomba de insulina pode ser um agravante ou um fator disparador. ${ }^{34}$

Dessa forma, compreender a relação que as mulheres com DM1 estabelecem com o seu corpo compõe uma dimensão 
importante a ser incorporada na prática assistencial a essa população. É a partir das suas concepções de beleza, autoimagem e autoestima que se pode identificar, prevenir e até mesmo tratar os TAs.

\section{CONSIDERAÇÕES FINAIS E IMPLICAÇÕES PARA A PRÁTICA}

Este estudo permite conhecer a relação que a mulher com DM1 estabelece com o seu corpo. Essa relação é pautada pela concepção social do belo feminino associado a padrões de magreza excessiva para o corpo da mulher que contribuem com a distorção da autoimagem, a insatisfação com o corpo e a diminuição da autoestima, compondo um cenário favorável ao surgimento de TAs, nesse caso, a diabulimia. A perda de peso é proporcionalmente associada ao aumento da aceitação social e justifica a utilização de métodos deletérios à saúde como a diminuição da dose ou a suspensão do uso de insulina.

Para essas mulheres, a não adesão ao tratamento não está associada à falta de conhecimento, ao contrário, aprenderam a manipular o uso da insulina com o objetivo de reduzir o peso. Também demonstraram a ciência de que tal prática ocasiona prejuízos à saúde no curto e longo prazo, mesmo assim, sinalizaram que, em determinados contextos, voltariam a manipular o tratamento.

Esta pesquisa reforça a influência das determinações sociais no processo saúde-adoecimento-cuidado e apresenta um cenário desafiador para os profissionais da saúde. Faz-se necessário radicalizar a abordagem interdisciplinar para o cuidado de pessoas com DM1, incorporando todas as dimensões do ser no momento de negociar as estratégias de cuidado com esses pacientes.

As limitações deste estudo estão relacionadas à incipiente produção científica sobre a temática, especialmente no contexto brasileiro e na dimensão subjetiva das mulheres com DM, o que dificulta a discussão e a comparação dos resultados. Aponta ainda a necessidade de aprofundar os estudos sobre os aspectos subjetivos que interferem nas práticas de autocuidado.

Os profissionais da saúde devem valorizar o desejo que as mulheres têm de perder peso e atuar conjuntamente em estratégias que não prejudiquem a saúde delas. A comunicação aberta, sem prejulgamentos ou condenações, é essencial para que essa relação se estabeleça de forma sincera e efetiva e, quem sabe, proporcione melhores resultados no cuidado à saúde.

\section{CONTRIBUIÇÕES DOS AUTORES}

Desenho do estudo. Letícia Braga Ribeiro. Claudia Maurício Pieper. Mônica Antar Gamba. Anderson da Silva Rosa.

Coleta de dados. Letícia Braga Ribeiro. Claudia Maurício Pieper. Anderson da Silva Rosa.

Análise de dados. Letícia Braga Ribeiro. Claudia Maurício Pieper. Mônica Antar Gamba. Anderson da Silva Rosa.

Interpretação dos resultados. Letícia Braga Ribeiro. Claudia Maurício Pieper. Giovana Andrade Frederico. Mônica Antar Gamba. Anderson da Silva Rosa.
Redação e revisão crítica do manuscrito. Letícia Braga Ribeiro. Claudia Maurício Pieper. Giovana Andrade Frederico. Mônica Antar Gamba. Anderson da Silva Rosa.

Aprovação da versão final do artigo. Letícia Braga Ribeiro. Claudia Maurício Pieper. Giovana Andrade Frederico. Mônica Antar Gamba. Anderson da Silva Rosa.

Responsabilidade por todos os aspectos do conteúdo e a integridade do artigo publicado. Letícia Braga Ribeiro. Claudia Maurício Pieper. Giovana Andrade Frederico. Mônica Antar Gamba. Anderson da Silva Rosa.

\section{EDITOR ASSOCIADO}

Stela Maris de Mello Padoin

\section{REFERÊNCIAS}

1. International Diabetes Federation. IDF diabetes atlas [Internet]. 9th ed. Brussels: IDF; 2019 [citado 2020 jul 26]. Disponível em: www. diabetesatlas.org

2. Andrade CJN, Alves CAD. Relationship between bullying and type 1 diabetes mellitus in children and adolescents: a systematic review. J Pediatr. 2019;95(5):509-18. http://dx.doi.org/10.1016/j.jped.2018.10.003. PMid:30391140.

3. Pinhas-Hamiel O, Hamiel U, Levy-ShragaY. Eating disorders in adolescents with type 1 diabetes: challenges in diagnosis and treatment. World $J$ Diabetes. 2015;6(3):517-26. http://dx.doi.org/10.4239/wjd.v6.i3.517 PMid:25897361.

4. Gagnon C, Aimé A, Bélanger C. Eating patterns in adolescents with type 1 diabetes: associations with metabolic control, insulin omission, and eating disorder pathology. PLoS One. 2015 jul;10(11):e0141386. http://dx.doi.org/10.1016/j.appet.2017.03.035. PMid:26529593.

5. Wisting L, Rø A, Skrivarhaug T, Dahl-Jørgensen K, Rø Ø. Disturbed eating, illness perceptions, and coping among adults with type 1 diabetes on intensified insulin treatment, and their associations with metabolic control. J Health Psychol. 2019 abr;3. http://dx.doi. org/10.1177/1359105319840688. PMid:30939939.

6. Quick VM, McWilliams R, Byrd-Bredbenner C. Case-control study of disturbed eating behaviors and related psychographic characteristics in young adults with and without diet-related chronic health conditions. Eat Behav. 2012 ago;13(3):207-13. http://dx.doi.org/10.1016/j. eatbeh.2012.02.003. PMid:22664398.

7. Wisting L, Skrivarhaug T, Dahl-Jørgensen K, Rø $\varnothing$. Prevalence of disturbed eating behavior and associated symptoms of anxiety and depression among adult males and females with type 1 diabetes. J Eat Disord. 2018 set;6(1):28. http://dx.doi.org/10.1186/s40337-018-0209-z. PMid:30214804.

8. Falcão MACRM. Diabetes, perturbações alimentares e imagem corpora em jovens adultos: estudo exploratório sobre a diabulimia [dissertação] Lisboa: Universidade de Lisboa; 2015.

9. Sociedade Brasileira de Diabetes. Diretrizes da Sociedade Brasileira de Diabetes 2019-2020. São Paulo: Editora Clannad; 2019.

10. Coleman SE, Caswell N. Diabetes and eating disorders: an exploration of 'Diabulimia'. BMC Psychol. 2020 set;8(1):101. http://dx.doi.org/10.1186/ s40359-020-00468-4. PMid:32967730.

11. Araia E, Hendrieckx C, Skinner T, Pouwer F, Speight J, King RM. Gende differences in disordered eating behaviors and body dissatisfaction among adolescents with type 1 diabetes: Results from diabetes MILES youth-Australia. Int J Eat Disord. 2017 ago;50(10):1183-93. http:// dx.doi.org/10.1002/eat.22746. PMid:28856699.

12. Mauss M. Sociologia e antropologia. 5. ed. São Paulo: Cosacnaify; 2015.

13. Claumann GA, Laus MF, Felden EPG, Silva DAS, Pelegrini A. Association between dissatisfaction with body image and health-related physical 
fitness among adolescents. Cien Saude Colet. 2019;24(4):1299-308. http://dx.doi.org/10.1590/1413-81232018244.17312017.PMid:31066833.

14. Rentz-Fernandes AR, Silveira-Viana M, De Liz CM, Andrade A. Autoestima imagem corporal e depressão de adolescentes em diferentes estados nutricionais. Rev Salud Publica. 2017 jan-fev; 19(1):66-72. http://dx.doi. org/10.15446/rsap.v19n1.47697. PMid:30137157.

15. Hosseini SA, Padhy RK. Body image distortion [Internet]. StatPearls 2020 [citado 2020 jul 26]. Disponível em: https://www.ncbi.nlm.nih.gov/ books/NBK546582/

16. Dourado CS, Fustinoni SM, Schirmer J, Brandão-Souza C. Body culture and meaning. J Hum Growth Dev. 2018;28(2):206-12. http://dx.doi. org/10.7322/jhgd.147240.

17. Rounsefell K, Gibson S, McLean S, Blair M, Molenaar A, Brennan L et al. Social media, body image and food choices in healthy young adults: a mixed methods systematic review. Nutr Diet. 2020 out:77(1):19-40. http://dx.doi.org/10.1111/1747-0080.12581. PMid:31583837.

18. Uzunian LG, Vitalle MSS. Social skills: a factor of protection against eating disorders in adolescentes. Cien Saude Colet. 2015 nov;20(11):3495508. http://dx.doi.org/10.1590/1413-812320152011.18362014. PMid:26602727.

19. Zhang J, Zhai Y, Feng XQ, Li WR, Lyu YB, Astell-Burt T et al. Gender differences in the prevalence of overweight and obesity, associated behaviors, and weight-related perceptions in a national survey of primary school children in China. Biomed Environ Sci. 2018;31(1):1-11. PMid:29409580.

20. Young-Hyman DL, Peterson CM, Fischer S, Markowitz JT, Muir AB, Laffel LM. depressive symptoms, emotion dysregulation, and bulimic symptoms in youth with type 1 diabetes. varying interactions at diagnosis and during transition to insulin pump therapy. J Diabetes Sci Technol. 2016 maio;10(4):845-51. http://dx.doi.org/10.1177/1932296816645118. PMid:27137457.

21. Thompson P. A voz do passado: história oral. 6. ed. Rio de Janeiro: Paz e Terra; 2002

22. Meihy JCSB, Holanda F. História oral: como fazer, como pensar. 3. ed São Paulo: Contexto; 2013

23. Meihy JCSB. Manual de história oral. 5. ed. São Paulo: Loyola, 2018.

24. Bloc LG, Nazareth ACP, Melo AKS, Moreira V. Transtorno de compulsão alimentar: revisão sistemática da literatura. Rev Psicol Saúde. 2019 jan-abr;11(1):3-17. http://dx.doi.org/10.20435/pssa.v11i1.617.

25. Heiman T, Olenik-Shemesh D. Perceived body appearance and eating habits: the voice of young and adult students attending higher education. Int J Environ Res Public Health. 2019 fev;16(3):451. http:// dx.doi.org/10.3390/ijerph16030451. PMid:30720728.
26. Staite E, Zaremba N, Macdonald P, Allan J, Treasure J, Ismail K et al 'Diabulima' through the lens of social media: a qualitative review and analysis of online blogs by people with Type 1 diabetes mellitus and eating disorders. Diabet Med. 2018 jun;35(10):1329-36. http://dx.doi. org/10.1111/dme.13700. PMid:29855073.

27. Holmström MR, Häggström M, Söderberg S. Being facilitators in a challenging context-school personnel's experiences of caring for youth with diabetes type 1. J Pediatr Nurs. 2018 ago;43:e114-9. http://dx.doi. org/10.1016/j.pedn.2018.08.007. PMid:30100521.

28. Pinhas-Hamiel O, Hamiel U, Greenfield Y, Boyko V, Graph-Barel C Rachmiel $\mathrm{M}$ et al. Detecting intentional insulin omission for weight loss in girls with type 1 diabetes mellitus. Int J Eat Disord. 2013 maio;46(8):819 25. http://dx.doi.org/10.1002/eat.22138. PMid:23674378.

29. Falcão MA, Francisco R. Diabetes, eating disorders and body image in young adults: an exploratory study about "diabulimia". Eat Weight Disord. 2017;22(4):675-82. http://dx.doi.org/10.1007/s40519-0170406-9. PMid:28597360.

30. Philippi ST, Cardoso MGL, Koritar P, Alvarenga M. Risk behaviors for eating disorder in adolescents and adults with type 1 diabetes. Rev Bras Psiquiatr. 2013 abr-jun;35(2):150-6. http://dx.doi.org/10.1590/15164446-2012-0780. PMid:23904020.

31. Almeida MC, Claudino DA, Grigolon RB, Fleitlich-Bilyk B, Claudino AM. Psychiatric disorders in adolescents with type 1 diabetes: a casecontrol study. Rev Bras Psiquiatr. 2018 jul-set;40(3):284-9. http://dx.doi. org/10.1590/1516-4446-2017-2259. PMid:29412336.

32. Young-Hyman D, de Groot M, Hill-Briggs F, Gonzalez JS, Hood K, Peyrot M. Psychosocial care for people with diabetes: a position statement of the American Diabetes Association. Diabetes Care. 2016 dez;39(12):212640. http://dx.doi.org/10.2337/dc16-2053. PMid:27879358.

33. Eisenberg MH, Lipsky LM, Dempster KW, Liu A, Nansel TR. I should but i can't: controlled motivation and self-efficacy are related to disordered eating behaviors in adolescents with type 1 diabetes. $J$ Adolesc Health. 2016 ago;59(5):537-42. http://dx.doi.org/10.1016/j. jadohealth.2016.06.008. PMid:27567063.

34. Prinz N, Bächle C, Becker M, Berger G, Galler A, Haberland H et al Insulin pumps in type 1 diabetes with mental disorders: real-life clinical data indicate discrepancies to recommendations. Diabetes Technol Ther. 2016 jan;18(1):34-8. http://dx.doi.org/10.1089/dia.2015.0180. PMid:26451621. 